\title{
The roles of deformation and orientation in heavy-ion collisions induced by light deformed nuclei at intermediate energy
}

\author{
X.G. Cao ${ }^{1,2}$ G.Q. Zhang, ${ }^{1,2}$ X.Z. Cai ${ }^{*}{ }^{1}$ Y.G. Ma, ${ }^{1}$ W. Guo, ${ }^{1}$ J.G. Chen,${ }^{1}$ W.D. Tian, ${ }^{1}$ D.Q. Fang, ${ }^{1}$ and H.W. Wang ${ }^{1}$ \\ ${ }^{1}$ Shanghai Institute of Applied Physics, Chinese Academy of Sciences, Shanghai 201800, China \\ ${ }^{2}$ Graduate School of the Chinese Academy of Sciences, Beijing 100049, China
}

(Dated: November 18, 2018)

\begin{abstract}
The reaction dynamics of axisymmetric deformed ${ }^{24} \mathrm{Mg}+{ }^{24} \mathrm{Mg}$ collisions have been investigated systematically by an isospin-dependent quantum molecular dynamics (IDQMD) model. It is found that different deformations and orientations result in apparently different properties of reaction dynamics. We revealed that some observables such as nuclear stopping power $(R)$, multiplicity of fragments, and elliptic flow are very sensitive to the initial deformations and orientations. There exists an eccentricity scaling of elliptic flow in central body-body collisions with different deformations. In addition, the tip-tip and body-body configurations turn out to be two extreme cases in central reaction dynamical process.
\end{abstract}

PACS numbers: 24.10.Cn, 25.70.Mn, 27.30.+t

Aligned experiments investigating how deformed ${ }^{165} \mathrm{Ho}$ target affects the total neutron reaction cross section from 2 to $125 \mathrm{MeV}$ 1 and scattering of $\alpha$ particles with $15 \leq E_{\alpha} \leq 23 \mathrm{MeV}$ [2] were carried out about forty years ago. The similar case occurs in nanoscale physics that the initial shape of hot droplets also has significant effects on fragmentation process in the molecular dynamics (MD) framework [3]. It is expected that deformed nuclei induced heavy-ion collisions (HICs) can result in obviously different properties of dynamical processes and final state observables compared with spherical cases. There are some reports about deformed $\mathrm{U}+\mathrm{U}$ collisions at relativistic and ultrarelativistic energies and it is suggested that deformed $\mathrm{U}+\mathrm{U}$ collisions are more likely to create quark-gluon plasma (QGP) and may resolve many outstanding problems [4 11]. The deformation effects on reaction cross section [12], elliptic flow 13] and heavyion fusion [14, 15] was also discussed recently. On the other hand, polarized target and beam have been widely applied related with spin effects in HICs [16] especially for the total and differential reaction cross section measurement of aligned deformed beams such as ${ }^{7} \mathrm{Li}$ [17] and ${ }^{23} \mathrm{Na}$ [18].

The spin polarized beams have been greatly promoted by projectile-fragmentation reactions recently [19], which brings large angular momentum into fragment spin. Not only the fragmentation process itself produces spin polarized fragments but also the produced spin orientated beam of deformed nuclei can provide valuable information on shape effects during collisions [20]. Therefore, it is very necessary to consider the degree of freedom of initial deformation since so many radioactive nuclei far from $\beta$-stability line may have large deformation. How-

*E-mail address: caixz@sinap.ac.cn ever, the knowledge about collisions induced by deformed nuclei is very poor especially at intermediate energy.

Due to the distinct differences in overlap region of deformed nuclei collisions, collisions of aligned deformed nuclei may give a clearer and deeper insight into the reaction mechanism such as the process of multifragmentation and the development of collective flow. The different orientational collisions also have the advantage in fixing the uncertain behavior of density dependent symmetry energy, which is an elementary open problem related not only to many problems in nuclear physics but also to a number of important issues in nuclear astrophysics 21]. Besides the advantage in studying reaction mechanism and dynamics, highly deformed nuclei induced reactions may also inspire exotic nuclei research such as halo 22] and cluster phenomena [23].

In this paper, ${ }^{24} \mathrm{Mg}+{ }^{24} \mathrm{Mg}$ collision system is used to investigate the initial deformation and orientation effects by a microscopic transport model: the IDQMD model 24], which was developed from the quantum molecular dynamics (QMD) model 25]. The main advantage of the QMD model is that it can explicitly treat the many body state of collision system. So it contains correlation effects to all orders and can treat the fragmentation and fluctuation of HICs well.

In this calculations, soft and hard nuclear equation of state (EOS) with the incompressibility of $K=200$ and $380 \mathrm{MeV}$, respectively, are used for comparison. Here the strength of symmetry potential $C_{\text {sym }}=32 \mathrm{MeV}$ 25] and experimental parameterized nucleon-nucleon cross section which is energy and isospin dependent are used. ${ }^{24} \mathrm{Mg}$ is approximately treated as a sharp-cutoff ellipsoid with large quadrupole deformation parameter: $\beta_{2}$ $=0.416$ 26]. For comparison, systematical calculations for tip-tip (body-body) collisions of ${ }^{24} \mathrm{Mg}+{ }^{24} \mathrm{Mg}$ with $\beta_{2}=0,0.05,0.1,0.2$ (all four cases with the same root mean-square radius) at different energies and impact pa- 


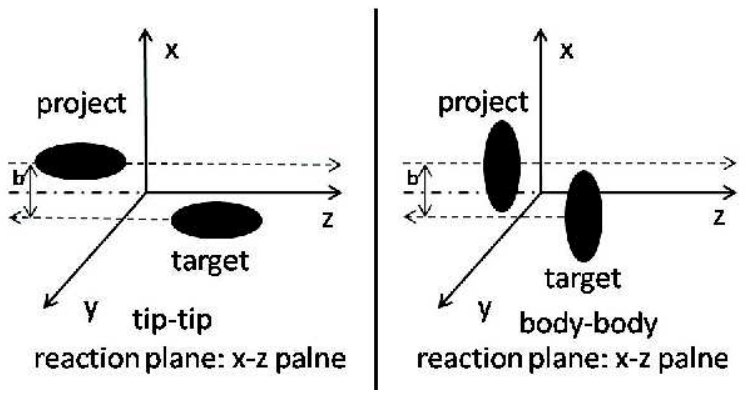

FIG. 1: Schematic representation of tip-tip and body-body collisions. In the coordinate system, $z$-direction is defined as the incident direction and the impact parameter $(b)$ is labeled as $x$-axis. Only $b$ to ${ }^{24} \mathrm{Mg}$ 's long axis in body-body collisions is considered.

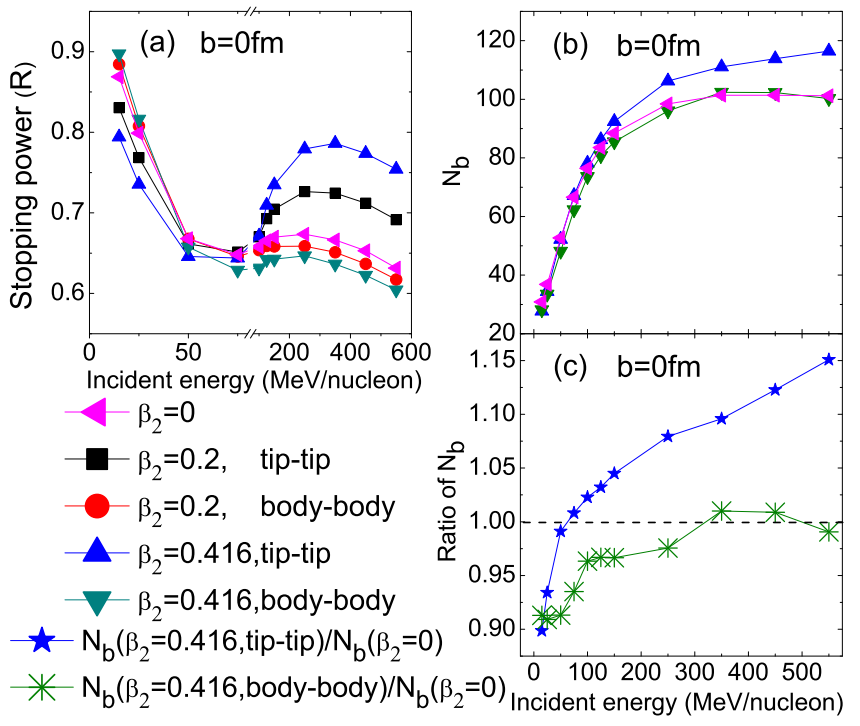

FIG. 2: (Color online) (a): $R$ and (b): total binary collision number $\left(N_{b}\right)$ as a function of incident energy at freeze-out time. (c): The ratio of non-spherical $N_{b}$ to spherical one.

rameters are carried out . The schematic plot of tip-tip and body-body collisions is illustrated by Fig. 1.

Firstly, we discuss the nuclear stopping power $(R=$ $\frac{2}{\pi} \sum_{i}^{A}\left|P_{i \perp}\right| / \sum_{i}^{A}\left|P_{i \|}\right|$, where A refers to the sum of projectile mass number and target mass number, $P_{i \perp}=\left(P_{i x}^{2}+P_{i y}^{2}\right)^{1 / 2}, P_{i \|}=P_{i z}$ in the c.m. reference system [27]) of different orientational collisions. $R$ can be used to describe the momentum dissipation and the degree of thermalization. Fig. 2 (a) shows that central body-body collisions lead to larger $R$ than central tiptip collisions below $50 \mathrm{MeV} /$ nucleon while the situation reverses when incident energies exceed $75 \mathrm{MeV} /$ nucleon. The more prolate ${ }^{24} \mathrm{Mg}$ is, more obvious differences appear. The spherical case lies between tip-tip and bodybody collisions at all calculated energies. The larger $R$ of tip-tip collisions at higher energy is in agreement with the result at $0.52 \mathrm{GeV} /$ nucleon by ART model [10]. However, the inversion of $R$ between tip-tip and body-body collisions is first observed. It reflects the different roles of the initial space configurations vs. energies.

It is known that the reaction mechanism at intermedi-

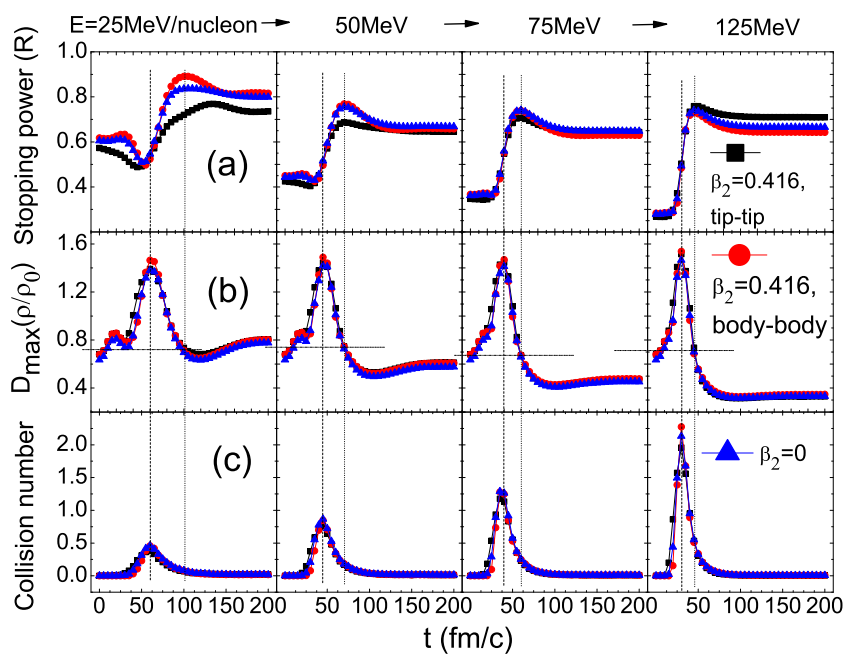

FIG. 3: (Color online) (a), (b) and (c) represent the time evolutions of $R$, maximal density $D_{\max }$ and $N_{b}$ in central collisions $(b=$ $0 \mathrm{fm})$, respectively. The long-dashed, short-dashed and dashdotted lines are drawn to mark the characteristic time of the collisions. The time structure of the $N_{b}$ is synchronous with the density evolution. Time evolution of $R$ show tip-tip, body-body and sphere-sphere collisions experience different touching, compressing and expanding processes from $t=0$ to freeze-out stage.

ate energy is dominated by mean field, binary collisions and Pauli blocking. Since the IDQMD model can treat the three components explicitly, it is very convenient to find out the factors which dominate the stopping power at different energies. As represented in Fig. 2 (b) and (c), tip-tip collision numbers are higher than body-body ones at all considered energies. It means that binary collision cannot be responsible for the inversion of $R$, while the mean field must play a very important role. Fig. 3 shows how mean field and binary collision take effect in dynamical process. The peak of density corresponds to the most intensive stopping process but the $R$ has not reached maximum. The departure between projectile-like and target-like continues contributing the nuclear stopping power. Through the different stopping behaviors of tip-tip and body-body collisions vs. energies, the time evolutions of $R$ show that when reaction proceeds more quickly, the larger stopping power can be achieved. So the stopping power can be regarded as a measurement of time scale of dynamical process as well as an observable of momentum dissipation.

Due to the larger projectile-target overlap region, body-body configurations build up stronger mean field, which lead to more violent one-body scattering. However, transparency effect of the nuclear medium becomes more and more important when incident energy rises. The tip-tip configurations are less transparent, which leads to larger two-body collisions and stopping power. Therefore, the underlying mechanics of the inversion of $R$ between tip-tip and body-body collisions is that body-body configurations build up stronger mean field 


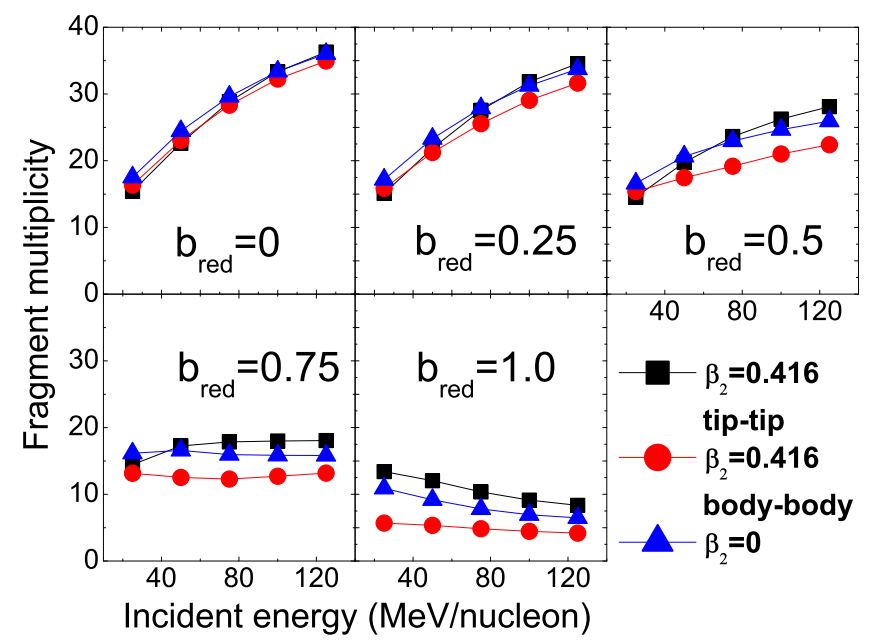

FIG. 4: (Color online) Energy dependence of fragment multiplicity of tip-tip, body-body and sphere-sphere collisions at different reduced impact parameters $\left(b_{\text {red }}=b / b_{\max }\right.$, where $b_{\max }$ refers to the maximal impact parameter for different cases).

at lower energies, where one-body scattering is predominant. Whereas two-body collisions become more important in tip-tip configurations at higher energies.

Since the IDQMD model can treat fragmentation of hot nuclei [28, 29] well. It is appropriate to investigate the fragmentation observables. As shown in Fig. 4, the fragment multiplicity has strong correlation with stopping power. Body-body collisions have minimal multiplicity at all impact parameters at higher energies while tip-tip collisions have the maximal one. So this behavior is consistent with that of stopping power at higher energies. It can also be seen from charge distributions in Fig. 5 that the tip-tip and body-body collisions are two extreme cases and the sphere-sphere collisions lie between them. Therefore, the fragment observables also confirm the similar picture indicated by $R$.

The Body-body collisions with $b=0 \mathrm{fm}$ will produce large collective motions due to the different initial geometry from spherical nuclei. Anisotropic flow method has been developed to measure the anisotropy of particle momentum space which related to the EOS and nuclear reaction dynamics 30 32. The azimuthal distribution of fragments can be expressed by Fourier expansion [33] $\frac{d N}{d \phi} \propto 1+2 \sum_{n=1}^{\infty} v_{n} \cos (n \phi)$, where $\phi$ is azimuthal angle between the transverse momentum of the particle and the reaction plane. The coefficient $v_{n}$ is defined as anisotropic flow parameter, among which $v_{2}$ denotes elliptic flow. It can be calculated in terms of single-particle averages: $v_{2}=\langle\cos (2 \phi)\rangle=\left\langle\frac{p_{x}^{2}-p_{y}^{2}}{p_{x}^{2}+p_{y}^{2}}\right\rangle$. Nucleon's $v_{2}$ induced by deformed $\mathrm{U}+\mathrm{U}$ collisions has been studied by ART model [5, 10] and optical Glauber model [13] at relativistic energies recently. It seems that the most central body-body collisions give rise to largest $v_{2}$ because of the strongest shadowing effect in the reaction plane [5]. Thus $v_{2}$ of central body-body collisions are most appro-

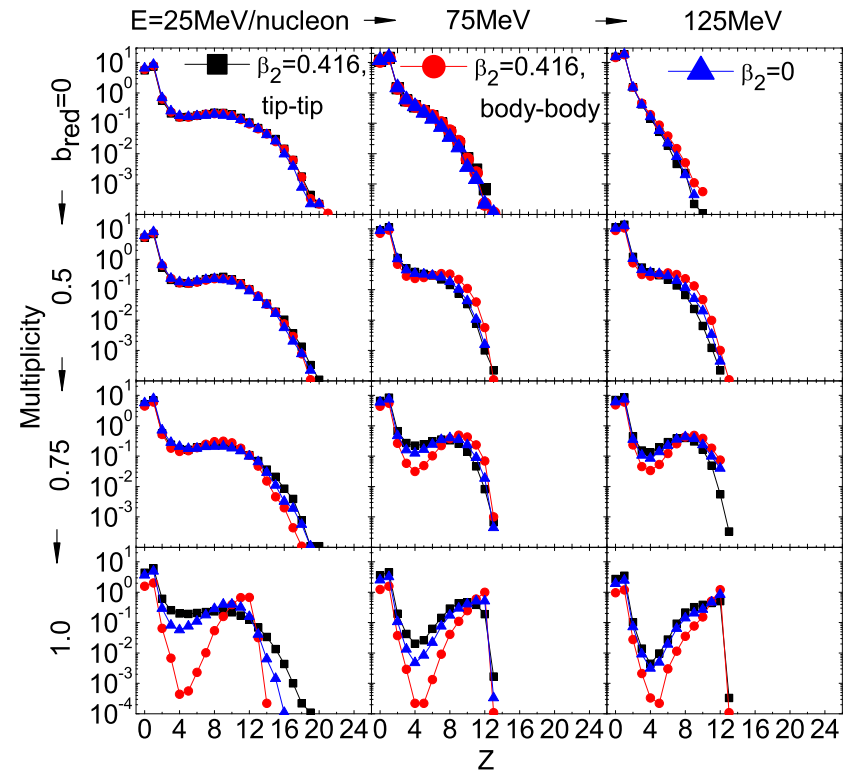

FIG. 5: (Color online) Charge distributions of tip-tip, body-body and sphere-sphere collisions at different $b_{\text {red }}$ and incident energies.

priate for investigating the EOS. However, $v_{2}$ developed from deformed nuclei collisions is unknown at intermediate energy and it's interesting to study their deformation and orientation effects.

$v_{2}$ of light fragments are shown in Fig. 6, in which the eccentricity $(\epsilon)$ is calculated by maximal geometry overlap region: $\epsilon=\sum_{i}\left(x_{i}^{2}-y_{i}^{2}\right) / \sum_{i}\left(x_{i}^{2}+y_{i}^{2}\right)$. Cental tip-tip and sphere-sphere collisions do not have obvious $v_{2}$ because of the transverse symmetry of overlap region while the $v_{2}$ of cental body-body collisions has non-zero value. The negative sign of $v_{2}$ at higher energies is in agreement with deformed $\mathrm{U}+\mathrm{U}$ collisions by ART model [5, 10]. The positive $v_{2}$ at lower energies and the alteration of sign for $v_{2}$ are first observed in central body-body collisions. At higher energies the violent two-body collisions in overlap region build the anisotropy pressure and it prompts fragments emission from in-plane preferential to out-of-plane preferential. The heavier fragments have larger $v_{2}$, which is consistent with ref. 34]. $v_{2}$ of central body-body collisions $\left(\beta_{2}=0.05,0.1,0.2,0.416\right)$ can be scaled together by $\epsilon$ from low energies to high energies. While scaled by the same $\epsilon$ amplitude as the deformed ${ }^{24} \mathrm{Mg}$ collisions, $v_{2}$ for mid-central spherical ${ }^{24} \mathrm{Mg}$ collisions shows different behaviors especially for higher energies. Therefore, the scaling of $v_{2}$ indicates that the geometric shapes of participants play an essential role in collective flow of central body-body collisions.

The energy excitation function of $v_{2}$ at midcentral sphere-sphere collisions varies from positive (inplane, rotational-like emission) to negative (out-of-plane, "squeeze-out" pattern) 31, 35]. This energy point is socalled transition energy, which is near $100 \mathrm{MeV} /$ nucleon 


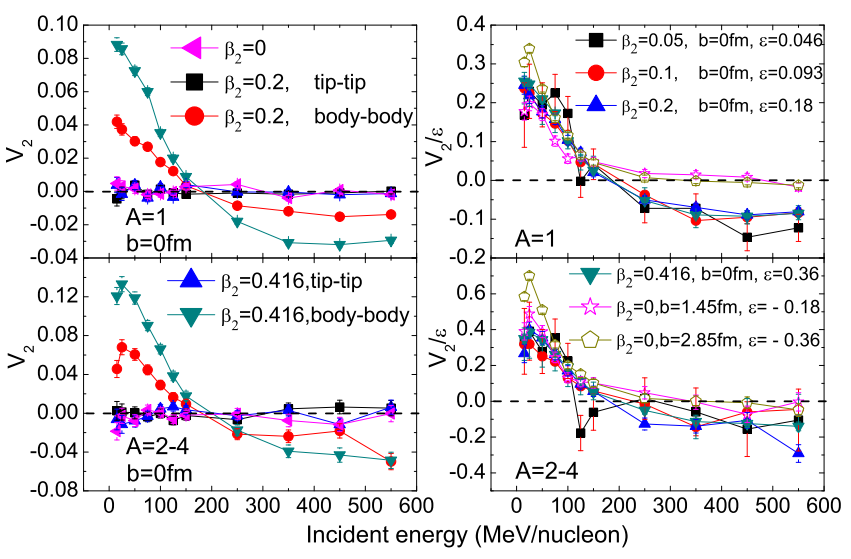

FIG. 6: (Color online) $v_{2}$ excitation function of light fragments at mid-rapidity $(-0.5<Y<0.5)$ of deformed and spherical collisions. The dashed lines are drawn to guide the eye. Left: $v_{2}$ in central collisions with $b=0 \mathrm{fm}$; Right: scaled $v_{2}$ with eccentricity $\epsilon$ in central body-body collisions and non-central spherical ${ }^{24} \mathrm{Mg}$ collisions. The spherical ${ }^{24} \mathrm{Mg}$ collisions with $b=1.45 \mathrm{fm}$ and $2.85 \mathrm{fm}$ have the same absolute value of $\epsilon$ as the deformed central ${ }^{24} \mathrm{Mg}$ collisions with $\beta_{2}=0.2$ and 0.416 , respectively.
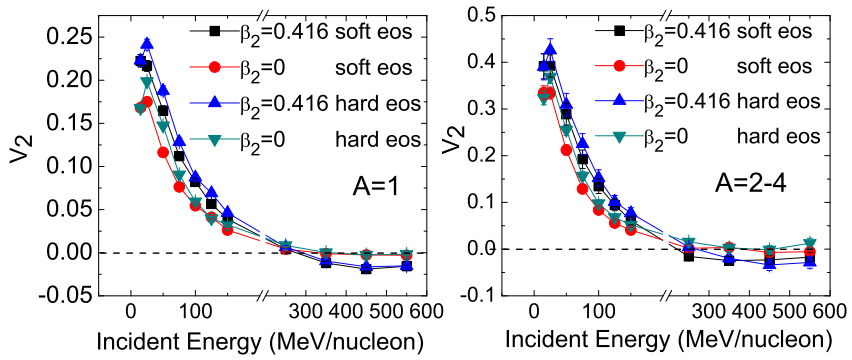

FIG. 7: (Color online) Average $v_{2}$ excitation function of light fragments at mid-rapidity $(-0.5<Y<0.5)$ for deformed and spherical collisions with soft and hard EOS. $v_{2}$ is averaged with $b$ from 0 to $b_{\max }$ for body-body and sphere-sphere collisions.

31]. For spherical collision system, there exist three competing components affecting the transition energy: (1) rotation of the compound system, (2) expansion of the hot and compressed participant zone, (3) shadowing of the colder spectator region 31]. Only the expansion survives in central spherical collisions [36, 37], which merely generates azimuthal symmetric flow. However, central body-body collisions have bulk transverse asymmetry overlap region and there is no rotation effect. Also the shadowing is different from mid-central collisions of spherical nuclei. Therefore, it provides an ideal tool to understand how the azimuthal pressure, expansion and flow development from the almond-shape overlap, which are all related with the extraction of the EOS. Average $v_{2}$ is shown in Fig. 7 with soft and hard EOS. Hard EOS enhances $v_{2}$ for both spherical and deformed collisions. Deformed configuration gives rise to larger $v_{2}$ than sphere-sphere configuration for both soft and hard EOS.

In summary, deformed ${ }^{24} \mathrm{Mg}+{ }^{24} \mathrm{Mg}$ collisions have been studied systematically by IDQMD model. The inversion of $R$ vs. energies between tip-tip and body-body collisions reflects the two different configurations play different roles on reaction dynamics. The fragment observables also show different behaviors for the two configurations. The sphere-sphere collisions lie between tip-tip and body-body collisions in nuclear stopping and fragmentation. Moreover, the excitation functions of $v_{2}$ for different deformed central body-body collisions can be scaled on a similar curve by eccentricity. $v_{2}$ averaged by impact parameter (collision configuration is represented by Fig. (1) in deformed collisions is stronger than that of spherical collisions for both soft and hard EOS. The large $v_{2}$ developed from cental body-body collisions have advantages in studying the EOS and transition energy. Tiptip collisions can be used to study the liquid-gas phase transition in finite nuclear systems due to the longer collision time. In addition, deformed nuclei collisions may have some implications on halo and cluster structure research. Therefore, the merits of collisions of deformed nuclei can shed light on the studies of both the nuclear structure and the reaction dynamics from low energies to relativistic energies.

This work is partially supported by National Natural Science Foundation of China under contract No.s 10775167, 10775168, 10979074, 10605036, 10875160, 10805067 and 10975174, Major State Basic Research Development Program in China under contract No. 2007CB815004, the Shanghai Development Foundation for Science and Technology under contract No. 09JC1416800 and the National Defence Innovation Foundation of Chinese Academy of Science under grants No. CXJJ-216.

[1] H. Marshak et al., Phys. Rev. Lett. 20, 554 (1968).

[2] D. R. Parks et al., Phys. Rev. Lett. 29, 1264 (1972).

[3] N. Komatsu and T. Abe, Phys. Rev. E 72, 021601 (2005).

[4] S. Das Gupta and C. Gale, Phys. Rev. C 62, 031901(R) (2000).

[5] B.-A. Li, Phys. Rev. C 61, 021903(R) (2000).

[6] E. V. Shuryak, Phys. Rev. C 61, 034905 (2000).

[7] U. Heinz and A. Kuhlman, Phys. Rev. Lett. 94, 132301 (2005).

[8] A. Kuhlman and U. Heinz, Phys. Rev. C 72, 037901 (2005).

[9] C. Nepali, G. Fai, and D. Keane, Phys. Rev. C 76, 051902(R) (2007).

[10] X.-F. Luo et al., Phys. Rev. C 76, 044902 (2007).

[11] H. Masui et al., Phys. Lett. B 679, 440 (2009).

[12] J. A. Christley and J. A. Tostevin, Phys. Rev. C 59, 2309 (1999).

[13] P. Filip, Phys. At. Nucl. 71, 1609 (2008).

[14] R. G. Stokstad et al., Phys. Rev. Lett. 41, 465 (1978).

[15] V. Y. Denisov and N. A. Pilipenko, Phys. Rev. C 76, 014602 (2007).

[16] D. Fick et al., Phys. Rep. 214, 1 (1992).

[17] K. H. Möbius et al., Phys. Rev. Lett. 46, 1064 (1981).

[18] N. M. Clarke et al., Phys. Rev. C 47, 660 (1993).

[19] K. Asahi, Prog. Part. Nucl. Phys. 46, 321 (2001). 
[20] Exploratory Workshop on Polarized Radioactive Beams and Polarized Targets, 6-7 March 2003, Strasbourg, Frances; http://wwwires.in2p3.fr/ires/workshops/polar03/.

[21] B.-A. Li et al., Phys. Rep. 464, 113 (2008).

[22] D. Q. Fang et al., Phys. Rev. C 76, 031601(R) (2007).

[23] M. Freer, Rep. Prog. Phys. 70, 2149 (2007).

[24] Y. G. Ma et al., Phys. Rev. C 73014604 (2006).

[25] J. Aichelin, Phys. Rep. 202, 233 (1991).

[26] G. A. Lalazissis et al., At. Data Nucl. Data Tables 71, 1 (1999).

[27] H. Ströbele et al., Phys. Rev. C 27, 1349 (1983).

[28] Y. G. Ma, Phys. Rev. Lett. 83, 3617 (1999).
[29] A. Bonasera et al., Rivista del Nuovo Cimento 23, 1 (2000).

[30] P. Danielewicz et al., Science 298, 1592 (2002).

[31] A. Andronic et al., Phys. Lett. B 612, 173 (2005).

[32] T. Z. Yan et al., Phys. Lett. B 638, 50 (2006).

[33] S. Voloshin et al., Z. Phys. C 70, 665 (1996).

[34] D. Brill et al., Z. Phys. A 355, 61 (1996).

[35] H. H. Gutbrod et al., Phys. Lett. B 216, 267 (1989).

[36] P. J. Siemens and J. O. Rasmussen, Phys. Rev. Lett. 42, 880 (1979).

[37] W. Reisdorf et al., Nucl. Phys. A 612, 493 (1997). 